\title{
Methicillin-Resistant Staphylococcus aureus Control: We Didn't Start the Fire, but It's Time to Put It Out
}

\author{
Carlene A. Muto, MD, MS
}

The prevalence of healthcare-associated infections (HAIs) caused by multidrug-resistant organisms has significantly increased over the past decade, according to the measurements of the Centers for Disease Control and Prevention, and will likely continue to climb. ${ }^{1,2}$ Currently, more than $70 \%$ of HAIs are caused by bacteria that are resistant to at least 1 of the drugs most commonly used to treat these infections. ${ }^{3}$ These HAIs have been associated with increased cost and mortality, compared with infections due to antibiotic-susceptible strains of the same bacterial species. ${ }^{4,5}$ Nevertheless, despite decades of studies confirming increased rates of adverse events related to these organisms and ongoing evidence of increased prevalence of multidrug-resistant organisms, ${ }^{2}$ as well as the emergence of new antimicrobial-resistant organisms, ${ }^{6}$ few coordinated effective efforts exist across the United States and most parts of the world to prevent MRSA transmission and reduce the number of MRSA HAIs. ${ }^{7}$ Despite evidence of efficacy for control of multidrug-resistant organisms with active surveillance cultures and barrier precautions ${ }^{8-10}$ and despite documented failure to control MRSA in studies lacking methods to fully identify the MRSA reservoir, ${ }^{8}$ most healthcare systems have not elected to implement methods to identify and contain MRSA, and so MRSA continues to spread and to colonize and infect patients.

Several studies in this issue of Infection Control and Hospital Epidemiology describe efforts to control methicillin-resistant S. aureus (MRSA). I comment here on 3 of them. ${ }^{11-13}$ Interestingly, the investigators in these 3 studies believe that colonized patients represented a significant portion of the MRSA reservoir and elected to implement a program of active surveillance cultures to better identify MRSA-colonized patients. In two of the studies, that of Salgado and Farr ${ }^{11}$ and that of Eveillard et al., ${ }^{12}$ barrier precautions were implemented for all patients identified as being MRSA colonized, as recommended by the recent Society for Healthcare Epidemiology of America (SHEA) guideline. ${ }^{8}$ The third study, by Sandri et al., ${ }^{13}$ discusses the utility of MRSA decolonization of the nares by administration of topical mupirocin therapy and chlor- hexidine baths to all patients identified as having MRSA colonization of the nares.

The study by Salgado and Farr ${ }^{11}$ reports the proportion of patients identified by active surveillance culture on admission as being MRSA colonized who later had an MRSA-positive clinical microbiology culture, the number and type of clinical microbiology cultures done, and the number and type of MRSA-positive clinical microbiology cultures. Their study demonstrates that only $15 \%$ of all patients identified as being colonized with MRSA on admission would have been so identified subsequently via clinical microbiology culture. When data for patients in the intensive care unit (ICU) and in other units (non-ICU patients) were analyzed separately, the investigators found that $21.4 \%$ of ICU patients had a subsequent MRSA-positive clinical microbiology culture during their hospital stay, whereas only $12.9 \%$ of non-ICU patients had an MRSA-positive clinical microbiology culture. This illustrates that ICU patients may be more likely to have MRSA identified via clinical microbiology culture than are non-ICU patients, but $78.6 \%$ of patients would have still been missed. If active surveillance culture was not done, the $85 \%$ of colonized patients unrecognized would have resulted in a total of 3,247 patient-days without isolation precautions in place (ie, nonisolated days), or between 1,936 and 2,373 nonisolated days, after adjusting for the time to MRSA active surveillance culture positivity. These findings are similar to data previously reported by Lucet et al., ${ }^{14}$ who reported that, without performance of active surveillance culture on admission for elderly patients, $76.2 \%$ of MRSA carriers would have been missed during their hospital stay, resulting in $81.1 \%$ of potential isolation days spent without isolation precautions in place. Similarly, the same investigator reported that $87.1 \%$ of MRSA carriers admitted to French ICUs would not have been identified as MRSA carriers if active surveillance culture was not being performed at admission. ${ }^{9}$ Data from these groups support ongoing use of active surveillance culture for MRSA on admission to rapidly identify both (1) the smaller proportion of patients with imported cases of MRSA colonization

Dr. Muto is from the Division of Hospital Epidemiology and Infection Control, University of Pittsburgh Medical Center, Presbyterian Campus, and the Infectious Diseases Epidemiology Research Unit, University of Pittsburgh School of Medicine and Graduate School of Public Health, Pittsburgh, Pennsylvania

Received January 20, 2006; accepted January 20, 2006; electronically published February 8, 2006. Infect Control Hosp Epidemiol 2006; 27:111-115

(C) 2006 by The Society for Healthcare Epidemiology of America. All rights reserved. 0899-823X/2006/2702-0001\$15.00. 
that would otherwise have been identified only later during hospitalization and (2) the majority of patients with imported cases who otherwise would never have been identified, so that barrier precautions could be initiated and the rate of transmission reduced.

The study by Eveillard et al. ${ }^{12}$ was undertaken during 1 year (from July 2002 through June 2003) in a 600-bed teaching hospital and compared the sensitivity of nasal, axilla, and rectal cultures alone and in combination (nasal and rectal cultures, axilla and rectal cultures, and all 3 types of culture) for identification of MRSA carriers on admission. Screening occurred within the first 48 hours after hospitalization for patients with high risk of MRSA carriage (defined as a history of MRSA carriage, hospitalization within the preceding year, transfer from another hospital, and/or the presence of chronic skin lesions) who were admitted to non-ICU wards and for all patients admitted to the ICU. Barrier precautions were implemented for all patients identified as being colonized with MRSA.

Screening methods were described as follows. Samples were streaked on mannitol salt agar containing an ofloxacin (10 $\mu \mathrm{g}$ ) disk and incubated for 48 hours at $30^{\circ} \mathrm{C}$. Only colonies recovered around the ofloxacin disk were further tested for oxacillin resistance. To my knowledge, ofloxacin resistance is not routinely used as a methodology for MRSA screening. Although some investigators have reported fluoroquinolone resistance as high as $95 \%-100 \%$ among MRSA isolates, the incidence of resistance to fluoroquinolones varies between clinical settings and countries. ${ }^{15}$ In settings where MRSA strains are not universally resistant to ofloxacin, MRSA isolates susceptible to ofloxacin would not be picked up using this screening method. As historic universal ofloxacin resistance in MRSA is not described at the French hospital where the study was done, we cannot be sure that all MRSA isolates were identified using this method. No standards exist that define the most effective microbiological media for MRSA screening, and no medium has been shown to be clearly superior; however, a prospective clinical trial tested 32 screening methods ${ }^{16}$ and found use of mannitol-salt agar containing lipovitellin with an oxacillin disk was associated with the highest yield. Mannitol-salt agar has been used for more than 50 years as a selective medium for the isolation of pathogenic staphylococci ${ }^{17,18}$; has been shown to be of value as a screening medium for MRSA, ${ }^{19,20}$ especially when used in combination with oxacillin ${ }^{21}$; and is recommended for MRSA screening by the American Society for Microbiology. ${ }^{22}$

Eveillard et al., ${ }^{12}$ like Salgado and Farr, ${ }^{11}$ report that clinical microbiology culture alone was not a reliable indicator of MRSA status. They found that $110(55.8 \%)$ of 197 patients newly identified as being colonized with MRSA would have been missed if clinical microbiology culture alone was used. Of the total of 123 patients found to be colonized with MRSA by means of screening cultures of specimens from multiple anatomical sites (nares, rectum, and axilla specimens), nares culture alone was more sensitive ( $73.2 \%$ of carriers identified) than rectal culture alone ( $46.9 \%$ of carriers identified) and axilla culture alone ( $25.21 \%$ of carriers identified); the rate of positive results increased when nares culture and axilla culture were used in combination (82.9\% of carriers identified) and increased to a greater degree when nares culture was used in combination with rectal culture $(91.9 \%$ of carriers identified). Other investigators have also reported that nares culture alone has been more sensitive than culture of specimens from other single body sites for detection of healthcareacquired MRSA colonization, ${ }^{23-25}$ but increased MRSA detection sensitivity has been reported when screening consisted of a combination of culture of specimens from the nares and one or more other anatomical sites ${ }^{24,25}$; therefore, MRSA active surveillance culture should always include samples from the anterior vestibule of the nose. ${ }^{8}$ Throat culture is also associated with a sensitivity equal to or greater than that of nasal cultures to detect MRSA in select patient populations, such as children. ${ }^{26,27}$ If used, the swab specimens from the throat and the nares can be plated onto the same agar plate, thus enhancing sensitivity without increasing culture cost.

The $27 \%$ of colonized patients who would have been missed with use of nares culture alone corresponded to 560 theoretical isolation days (TIDs); 431 TIDs would have been missed without rectal-culture screening, and 99 TIDs would have been missed without axilla-culture screening. This study noted that screening by nares culture alone would have missed $44 \%$ of colonized surgical patients, so perhaps population-specific screening strategies can be deployed and an additional anatomical site sampled for select populations. Other studies have not reported higher sensitivity (ie, greater than 95\%) with use of nares cultures alone. ${ }^{23}$ This dramatically differs from the results of Eveillard et al. ${ }^{22}$ Perhaps the variance can be explained by their microbiologic methods or the introduction of community-acquired MRSA into their hospital, because these strains may not be as readily identified with nares culture, ${ }^{28}$ and data from antibiograms or genotyping are not reported for the isolates identified with active suryeillance culture.

The ultimate goal of screening is to identify the reservoir of colonized patients. Clearly, culturing samples from more anatomical sites offers an advantage, but at a significant cost, and hospitals using only nares culture have reported successful control of MRSA. ${ }^{8}$ If resources were unlimited, culturing all sites for all patients would identify more MRSAcolonized patients, but exactly how many more is yet to be determined. Given the limited number of dollars available and the long list of worthy patient-safety initiatives, it seems reasonable to limit the anatomical sites cultured and to target high-risk populations for types of culture associated with the highest yield until better and more cost-effective screening methods are available.

Ideal screening methods should be rapid, sensitive, and inexpensive. Use of assays with greater sensitivity could eliminate the need for culture of additional anatomical sites. Recently, 2 new screening options became available, both reported to have greater sensitivity. In November 2004, a new selective and dif- 
ferential chromogenic medium was approved by the US Food and Drug Administration (BBL CHROMagar MRSA; BD Diagnostics). A multicenter study found that culture using this medium had high sensitivity and specificity, compared with other standards, including mecA polymerase chain reaction ( $95.1 \%$ sensitivity and $98.1 \%$ specificity)..$^{29}$ Eighty-six percent of MRSA isolates were identified within 24 hours of plating the specimen, and the remaining $14 \%$ within 48 hours. The second new option is a rapid polymerase chain reaction assay (IDI-MRSA; GeneOhm Sciences, BD Diagnostics). ${ }^{30}$ This is the only rapid molecular-based assay (ie, the procedure time for the assay is less than 2 hours) approved by the US Food and Drug Administration and Health Canada for the direct detection of MRSA from a nasal specimen, and it is reported to have a high sensitivity but at a significant increase in price. It is, however, important to note that running this assay throughout a 24 -hour period is not practical in a clinical laboratory setting, and so turnaround time for results may vary. This MRSA detection tool is currently being evaluated at Northwestern University Hospital (Chicago, IL). ${ }^{31}$

The study in this issue by Sandri et al. ${ }^{13}$ reports that MRSA infections in a 13-bed "general" ICU were significantly reduced ( $\chi^{2}$ for trend $=16.4, P=.00005$ ) during a 5-year open-label trial of treatment with $2 \%$ mupirocin ointment, which was applied to the nasal mucosa 3 times daily for 5 days, and chlorhexidine baths (concentration unspecified), given once daily for 3 days. These treatments were given to all patients found to have nasal MRSA colonization by means of surveillance cultures performed on admission and weekly thereafter. Mupirocin susceptibility of MRSA isolates was reported only for year 5 of the study (2003), and resistant strains were reported in $5 \%$ of patients.

The first question that needs to be addressed is the utility of mupirocin treatment for MRSA eradication. Selective suppression and/or eradication of MRSA carriage using mupirocin has been used adjunctively to help control spread of MRSA, ${ }^{38}$ but successful eradication is sometimes transient. Doebbeling et $\mathrm{al} .{ }^{39}$ reported that $26 \%$ of patients were recolonized within 4 weeks after decolonization, and $48 \%$ of patients who underwent an eradication protocol were culture positive for MRSA after 6 months. ${ }^{40}$ Use of mupirocin to eradicate MRSA colonization was studied in a randomized, controlled trial involving colonized hospital patients in Switzerland. All patients were treated with chlorhexidine baths, and half were randomized to receive mupirocin nasal treatment in addition. The group that received both chlorhexidine and mupirocin were treated for 5 days, and MRSA were eradicated in only $25 \%$ of the patients, whereas in the group that received chlorhexidine baths and placebo nasal ointment, MRSA were eradicated in $18 \%$; this suggests there was no significant additive benefit from the mupirocin treatment. ${ }^{41}$ Higher eradication rates were achieved when patients were given systemic therapy with a regimen containing rifampin and at least 1 other systemic drug to which MRSA was susceptible, and indwelling devices and other foreign bodies were removed or replaced halfway through the treatment course. ${ }^{34}$ Colonization at sites other than the nose was, as might be expected, a risk factor for persistent carriage despite the therapy. ${ }^{42}$

Although mupirocin-resistant strains had not yet spread through the Brazilian population studied by Sandri et al., ${ }^{13}$ it is worth noting that mupirocin has been shown to be ineffective for reliable eradication of mupirocin-resistant strains, ${ }^{43}$ although one study suggested that it might sometimes be useful in that situation. ${ }^{44}$ Multiple studies have reported the arrival (or development) and spread of mupirocinresistant strains in settings where mupirocin is in frequent use, ${ }^{45,46}$ including multiple reports of resistant strains spreading in Brazilian hospitals. ${ }^{47,48}$ Mupirocin has also rarely been associated with toxic epidermal necrolysis after topical intranasal application, so a decision to apply it with great frequency would have to take this risk into account. ${ }^{49}$ One recent meta-analysis of studies reporting the results of therapy for eradication of MRSA reported much conflicting evidence and concluded, "There is insufficient evidence to support use of topical or systemic antimicrobial therapy for eradicating nasal or extranasal MRSA. There is no demonstrated superiority of either topical or systemic therapy, or of combinations of these agents. Potentially serious adverse events and development of antimicrobial resistance can result from therapy. ${ }^{.50}$

Another issue not addressed by Sandri et al. ${ }^{13}$ was the lack of follow-up for decolonized patients. Hospital-wide rates of MRSA HAI were not reported; perhaps eradication with mupirocin merely delayed the onset of MRSA infection, and so it would not be reflected in the reported rate of MRSA HAIs in the ICU. Because recolonization has been reported, ${ }^{39,40}$ it would have been useful to follow up the decolonized cohort longitudinally and assess MRSA colonization and infection status.

Aggregate MRSA data are reported for the study period: respiratory infections accounted for $63 \%$ of all MRSA HAIs, bloodstream infections for $21 \%$, and skin and soft-tissue infections for $9 \%$. Since data on MRSA HAIs by body site are not reported by year, it is impossible to assess the reductive contribution of each infection type or to determine whether a specific infection initiative might have been responsible for the decrease of that type of HAI. The approach suggested by Sandri et al. ${ }^{13}$ would have to be considered with caution and would require confirmation by multiple other investigators in other populations, as the authors conclude, before this approach could be recommended for routine therapy.

The recent SHEA guideline recommended routine use of active surveillance culture to identify MRSA-colonized patients and use of isolation precautions for such patients, and it cited scores of studies reporting significantly reduced rates of colonization and infection after this intervention, many of which did not use eradication therapy for colonization. ${ }^{8}$ These interventions, which were very effective for MRSA control, involved ICUs ${ }^{51,52}$ entire hospitals, ${ }^{53}$ and settings where it is endemic and/or epidemic, so it seems that control may be achievable without resorting to routine and/or widespread 
eradication therapy. In one such study, the number of MRSA infections was reduced by more than $90 \%$ during a 4 -year period in a medical ICU by use of active surveillance culture and contact precautions but not eradication therapy. ${ }^{51}$ In another such study, the number of cases of MRSA bacteremia was reduced by $75 \%$ during the 16 months after phasing in use of active surveillance culture and contact precautions. ${ }^{52}$ It should be noted that one recent study suggested adverse effects of patient isolation (ie, fluid and electrolyte disorders, falls, and pressure sores), but the authors of this nonrandomized study noted that there were no significantly higher rates of adverse events involving diagnostic, operative, anesthetic, or medical procedures or drugs, nor was there a significant increase in mortality. They also emphasized that their findings would require confirmation in follow-up studies. ${ }^{54}$ Active detection and patient isolation have been used successfully for centuries as a strategy for containing the spread of contagious infections. This strategy clearly worked to contain emerging infections like severe acute respiratory syndrome (SARS) and nosocomial smallpox, as was emphasized by Alfred Sommer, Dean Emeritus, Johns Hopkins Bloomberg School of Public Health, in the plenary session of the 2004 meeting of SHEA. And it has worked to control MRSA infections, which cause morbidity in hundreds of thousands of US hospital patients each year and likely kill more than 10,000 patients. This is an important problem and deserves effective control measures.

So there has been widespread failure to control antibioticresistant nosocomial infections due to pathogens such as MRSA. It's time for action; patient safety can't wait. The recent SHEA guideline on control of MRSA and vancomycinresistant enterococci recommends use of active surveillance cultures to fully define the population harboring multidrugresistant organisms, coupled with use of appropriate barrier precautions for colonized and infected patients and routine hand hygiene before and after all contact with these patients. ${ }^{8}$ This combination of identification and containment is the most effective method documented to control multidrugresistant organisms and is recommended by the Centers for Disease Control and Prevention for control of other important (yet less prevalent) pathogens, such as the SARS coronavirus and vancomycin-resistant $S$. aureus. ${ }^{6,55}$ The panelists at a recent patient safety conference ("Leadership Issues: Eliminating the Spread of MRSA," sponsored by the Maryland Patient Safety Center and held in Linthicum, MD, in January 2005) all concurred and supported global implementation of active surveillance culture and barrier precautions across the United States, and recommended that healthcare facilities should intensify interventions to prevent spread of multidrug-resistant organisms when there is evidence of continuing transmission within a facility. It now seems that control plans, including use of active surveillance cultures, are inescapable. It is time to take action, make patient safety a priority, and put the fire out.
Address reprint requests to Carlene A. Muto, MD, MS, University of Pittsburgh Medical Center (UPMC-P), 1215 Kaufmann Building, Pittsburgh, PA 15213 (mutoca@msx.upmc.edu).

\section{REFERENCES}

1. Centers for Disease Control and Prevention. Proportion of healthcareacquired S. aureus infections resistant to oxacillin (MRSA) among ICU patients, 1989-2003. Vol. 2004. Available at: http://www.cdc.gov/ncidod/ dhqp/ar_mrsa.html. Accessed January 25, 2006.

2. Centers for Disease Control and Prevention. National Nosocomial Infections Surveillance (NNIS) system report, data summary from January 1992 through June 2004, issued October 2004. Am J Infect Control 2004; 32:470-485.

3. Centers for Disease Control and Prevention. Campaign to prevent antimicrobial resistance in healthcare settings: why a campaign? 2001. Available at: http://www.cdc.gov/drugresistance/healthcare/problem.htm. Accessed January 27, 2006.

4. Cosgrove SE, Sakoulas G, Perencevich EN, Schwaber M), Karchmer AW, Carmeli Y. Comparsion of mortality associated with methicillin-resistant and methicillin-susceptible Staphylococcus aureus bactermia: a meta-analysis. Clin Infect Dis 2003; 36:53-59.

5. Engemann J, Carmeli Y, Cosgrove SE, et al. Adverse clinical and economic outcomes attributable to methicillin resistance among patients with Staphylococcus aureus surgical site infections. Clin Infect Dis 2003; 36: 592-598.

6. Centers for Disease Control and Prevention (CDC). Vancomycin-resistant Staphylococcus aureus-New York, 2004. MMWR Morb Mortal Wkly Rep 2004; 53:322-323.

7. Muto CA. Why are antibiotic-resistant nosocomial infections spiraling out of control? Infect Control Hosp Epidemiol 2005; 26:10-12.

8. Muto CA, Jernigan JA, Ostrowksy BE, et al. SHEA Guideline for preventing nosocomial transmission of multidrug-resistant strains of Staphylococcus aureus and Enterococcus. Infect Control Hosp Epidemiol 2003; 24:362-386.

9. Lucet JC, Chevert S, Durand-Zaleski I, Chastang C, Regnier B. Multicenter Study Group. Prevalence and risk factors for carriage of methicillin-resistant Staphylococcus aureus at admission to the intensive care unit: results of a multicenter study. Arch Intern Med 2003; 163:181-188.

10. Boyce JM, Havill NL, Kohan C, Dumigan DG, Ligri CE. Do infection control measures work for methicillin-resistant Staphylococcus aureus? Infect Control Hosp Epidemiol 2004; 25:395-401.

11. Salgado CD, Farr BM. What proportion of hospital patients colonized with methicillin-resistant Staphylococcus aureus are identified by clinical microbiological cultures? Infect Control Hosp Epidemiol 2006; 27:116-121 (in this issue).

12. Eveillard M, de Lassence A, Lancien E, Barnaud G, Ricard JD, Joly-Guillou ML. Evaluation of a strategy of screening multiple anatomical sites for methicillin-resistant Staphylococcus aureus at admission to a teaching hospital. Infect Control Hosp Epidemiol 2006; 27:181-184 (in this issue).

13. Sandri AM, Dalarosa MG, de Alcântara LR, da Silva Elias L, Zavascki AP. Reduction in incidence of nosocomial methicillin-resistant Staphylococcus aureus (MRSA) infection in an intensive care unit: role of treatment with mupirocin ointment and chlorhexidine baths for nasal carriers of MRSA. Infect Control Hosp Epidemiol 2006; 27:185-187 (in this issue).

14. Lucet JC, Grenet K, Armond-Lefevre L, et al. High prevalence of carriage of methicillin-resistant Staphylococcus aureus at hospital admission in elderly patients: implications for infection control strategies. Infect Control Hosp Epidemiol 2005; 26:121-126.

15. Goldstein FW, Acar JF. Epidemiology of quinolone resistance: Europe and North and South America. Drugs 1995; 49:36-42.

16. Safdar N, Narans L, Gordon B, Maki DG. Comparison of culture screening methods for detection of nasal carriage of methicillin-resistant Staphylococcus aureus: a prospective study comparing 32 methods. J Clin Microbiol 2003; 41:3163-3166. 
17. Blair EB, Emerson JS, Tull AH. A new medium, salt mannitol plasma agar, for the isolation of Staphylococcus aureus. Am J Clin Pathol 1967; 47:30-39.

18. Chapman GH. The significance of sodium chloride in studies of staphylococci. J Bacteriol 1945; 50:201-203.

19. La Zonby JG, Starzyk MJ. Screening method for recovery of methicillinresistant Staphylococcus aureus from primary plates. J Clin Microbiol 1986; 24:186-188.

20. Merlino J, Gill R, Robertson G]. Application of lipovitellin-salt-mannitol agar for screening, isolation, and presumptive identification of Staphylococcus aureus in a teaching hospital. J Clin Microbiol 1996; 34:3012-3015.

21. van Enk RA, Thompson KD. Use of a primary isolation medium for recovery of methicillin-resistant Staphylococcus aureus. J Clin Microbiol 1992; 30:504-505.

22. Gorss EB. Prospective, focused surveillance for oxacillin-resistant Staphylococcus aureus. In: Isenberg IHD, ed. Clinical microbiology procedures handbook. Washington, DC: American Society for Microbiology; 1992: 11.15.1-11.15.2.

23. Singh $K$, Gavin PJ, Vescio $T$, et al. Microbiologic surveillance using nasal cultures alone is sufficient for detection of methicillin-resistant Staphylococcus aureus isolates in neonates. J Clin Microbiol 2003; 41:2755-2757.

24. Sanford MD, Widmer AF, Bale MJ, Jones RN, Wenzel RP. Efficient detection and long-term persistence of the carriage of methicillin-resistant Staphlyococcus aureus. Clin Infect Dis 1994; 19:1123-1128.

25. Papia G, Louie M, Tralla A, Johnson C, Collins V, Simor AE. Screening high-risk patients for methicillin-resistant Staphylococcus aureus on admission to the hospital: is it cost effective? Infect Control Hosp Epidemiol 1999; 20:473-477.

26. Shahin R, Johnson IL, Jamieson F, McGeer A, Tolkin J, Ford-Jones EL. Methicillin-resistant Staphylococcus aureus carriage in a child care center following a case of disease. Arch Pediatr Adolesc Med 1999; 153:864-868.

27. Sa-Leau R, Sanches IS, Couto I, Alves CR, de Lencastre H. Low prevalence of methicillin-resistant strains among Staphylococcus aureus colonizing young and healthy members of the community in Portugal. Microbial Drug Resistance 2001; 7:237-245.

28. Rihn JA, Michaels MG, Harner CD. Community-acquired methicillinresistant Staphylococcus aureus: an emerging problem in the athletic population. Am J Sports Med 2005; 33:1924-1929.

29. Flayhart D, Hindler JF, Bruckner DA, et al. Multicenter evaluation of BBL CHROMagar MRSA medium for direct detection of methicillinresistant Staphylococcus aureus from surveillance cultures of the anterior nares. J Clin Microbiol 2005; 43:5536-5540.

30. Warren DK, Liao RS, Merz LR, Eveland M, Dunne WM, Jr. Detection of methicillin-resistant Staphylococcus aureus directly from nasal swab specimens by a real-time PCR assay. J Clin Microbiol 2004; 42:5578-5581.

31. Check W. Border patrol: MRSA admission screening. CAP Today 2005; 19. Available at: http://www.cap.org/apps/docs/cap_today/cover_stories/ 1005_MRSA_Admission_Screening.html. Accessed January 30, 2006.

32. Back NA, Linnemann CC Jr, Staneck JL, Kotagal UR. Control of methicillin-resistant Staphylococcus aureus in a neonatal intensive-care unit: use of intensive microbiologic surveillance and mupirocin. Infect Control Hosp Epidemiol 1996; 17:227-231.

33. Struelens MJ, Ronveaux O, Jans B, Mertens R, the Groupement pour le Depistage, 'Etude et la Prevention des Infections Hospitalieres. Methicillin-resistant Staphylococcus aureus epidemiology and control in Belgian hospitals, 1991 to 1995. Infect Control Hosp Epidemiol 1996; 17:503-508.

34. Karchmer TB, Jernigan JA, Durbin BM, Simonton BM, Farr BM. Eradication of methicillin-resistant $S$. aureus (MRSA) colonization with different regimens. Program and Abstracts of the Ninth Annual Meeting of The Society for Healthcare Epidemiology of America- San Francisco, CA, April 18-20 1999. Abstract 65, p. 42.

35. Kotilainen $P$, Routamaa $M$, Peltonen $R$, et al. Eradication of methicillinresistant Staphylococcus aureus from a health center ward and associated nursing home. Arch Intern Med 2001; 161:859-863.

36. Kluytmans J, Mouton JW. IJzerman EPF, et al. Reduction of surgicalsite infections in cardiothoracic surgery by elimination of nasal carriage of Staphylococcus aureus. Infect Control Hosp Epidemiol 1996; 17:780-785.
37. Kluytmans JA, Manders MJ, van Bommel E, Verbrugh H. Elimination of nasal carriage of Staphylococcus aureus in hemodialysis patients. Infect Control Hosp Epidemiol 1996; 17:793-797.

38. Weber S, Herwaldt LA, Mcnutt LA, et al. An outbreak of Staphylococcus aureus in a pediatric cardiothoracic surgery unit. Infect Control Hosp Epidemiol 2002; 23:77-81.

39. Doebbeling BN, Breneman DL, Neu HC, et al. Elimination of Staphylococcus aureus nasal carriage in health care workers: analysis of six clinical trials with mupirocin calcium ointment. The Mupirocin Collaborative Study Group. Clin Infect Dis 1993; 17:466-474.

40. Doebbeling BN, Regan DR, Pfaller MA, Houston AK, Hollis RJ, Wenzel RP. Long term efficacy of intranasal mupirocin ointment. A prospective cohort study of Staphylococcus aureus carriage. Arch Intern Med 1994; 154:1505-1508.

41. Harbarth S, Dharan S, Liassine N, Herrault P, Auckenthaler R, Pittet D. Randomized, placebo-controlled, double-blind trial to evaluate the efficacy of mupirocin for eradicating carriage of methicillin-resistant Staphylococcus aureus. Antimicrob Agents Chemother 1999; 43:1412-1416.

42. Harbarth S, Dharan S, Liassine N, Herrault P, Auckenthaler R, Pittet D. Risk factors for persistent carriage of methicillin-resistant Staphylococcus aureus. Clin Infect Dis 2000; 31:1380-1385.

43. Walker ES, Vasquez JE, Dula R, Bullock H, Sarubbi FA. Mupirocinresistant, methicillin-resistant Staphylococcus aureus: does Mupirocin remain effective? Infect Control Hosp Epidemiol 2003; 24:342-346.

44. Semret M, Miller MA. Topical mupirocin for eradication of MRSA colonization with mupirocin-resistant strains. Infect Control Hosp Epidemiol 2001; 229:578-580.

45. Vasquez JE, Walker ES, Franzus BW, Overbay BK, Regan DR, Sarubbi FA. The epidemiology of mupirocin resistance among methicillin-resistant Staphylococcus aureus at a Veterans' Affairs hospital. Infect Control Hosp Epidemiol 2000; 21:459-464.

46. Miller MA, Dascal A, Portnory J, Medelson J. Development of mupirocin resistance among methicillin-resistant Staphyloccus aureus after widespread use of nasal mupirocin ointment. Infect Control Hosp Epidemiol 1996; 17:811-813.

47. Netto dos Santos K, de Souza FL, Gontijo Filho P. Emergence of highlevel mupirocin resistance in methicillin-resistant Staphylococcus aureus isolated from Brazilian university hospitals. Infect Control Hosp Epidemiol 1996; 17:813-816.

48. Vivoni AM, Santos KR, de-Oliveira MP, et al. Mupirocin for controlling methicillin-resistant Staphylococcus aureus: lessons from a decade of use at a university hospital. Infect Control Hosp Epidemiol 2005; 26:662-667.

49. Praz SM, de Torrente A, Zender H, Schmied E, Schleppy CA, Genne D. Toxic epidermal necrolysis after topical intranasal application of mupirocin. Infect Control Hosp Epidemiol 2003; 24:459-460.

50. Loeb M, Main C, Walker-Dilks C, Eady A. Antimicrobial drugs for treating methicillin-resistant Staphylococcus aureus colonization. Cochrane Database Syst Rev 2003; 2:CD003340.

51. Blank MK, Haas L, Donahoe M, Kramer P, Muto CA. Sustained effect in reducing methicillin-resistant Staphylococcus aureus (MRSA) hospital acquired infections (HAIs) using active MRSA surveillance cultures (MSC) - Three-year follow-up. Paper presented at: 15th Annual Society for Healthcare Epidemiology of America Conference, Los Angeles, California, 2005. Abstract 22.

52. Huang SS, Yokoe DS, Rego VH, et al. Impact of ICU surveillance for MRSA on bacteremia. Paper presented at: 43rd Annual Meeting of The Infectious Diseases Society of America, San Francisco, CA, October 6-9 2005.

53. Thompson R CI, Wenzel R,. Epidemiology of nosocomial infections caused by methicillin-resistant Staphylococcus aureus. Ann Intern Med 1982; 97:309-317.

54. Stelfox HT, Bates DW, Redelmeier DA. Safety of patients isolated for infection control. JAMA 2003; 290:1899-1905.

55. Centers for Disease Control and Prevention (CDC). Public health guidance for community-level preparedness and response to SARS. 2004. Available at: http://www.cdc.gov/ncidod/sars/guidance/index.htm. Accessed January 27, 2006. 